\title{
An anisotropic damage model of elasticity based on a series coupling method
}

\author{
Linlin Sun*, Weiping Hu**, Qingchun Meng*** \\ *BeiHang University, Beijing 100191, China, E-mail: linscent.sun@gmail.com \\ **BeiHang University, Beijing 100191, China, E-mail: huweiping@buaa.edu.cn \\ ***BeiHang University, Beijing 100191, China, E-mail: Qcmeng@buaa.edu.cn
}

cross' $^{\text {ref }}$ http://dx.doi.org/10.5755/j01.mech.22.1.12352

\section{Introduction}

Since Kachanov [1] firstly introduced a scalar damage concept to describe creep of metals in 1958, continuum damage mechanics has been applied to different materials, such as concrete, geological materials, polymers, composites and other materials, and to a wide variety of damage phenomena including elastic-plastic damage, elastic-brittle damage, fatigue damage, dynamic and spall damage, etc [2].

In the early studies, isotropic damage theory which adopts a scalar variable defined in terms of the reduction in cross-sectional area $[1,3]$ to describe the degradation of materials due to the development of micro-voids or micro-cracks. Then some new scalar damage variables in terms of the reduction in the elastic modulus or elastic stiffness $[4,5]$, the shear modulus, the bulk modulus, and the Poisson's ratio [6] are proposed. However, it has been shown that the damage path is somewhat too restrictive and not universal even among isotropic damage processes when using only one damage variable to describe the isotropic damage [7-9]. Two damage variables must be adopted in order to describe accurately and consistently the special case of isotropic damage. Cauvin and Testa [8] used two scalar isotropic damage parameters $D_{1}$ and $D_{2}$ that do not have simple physical meanings, Tang et al. [9] proposed different groups of two scalar isotropic damage parameters, for example, $D_{E}$ and $D_{v}$ that represent the reduction of the elastic modulus and the Poisson's ratio, respectively.

Because of its scalar nature, the evolution equations of isotropic damage are easy to handle. Lemaitre [10] pointed out that the assumption of isotropic damage is often sufficient to give good prediction of the loading capacity, the number of cycles or the time to local failure in structural components. However, experiments have shown that anisotropic damage would develop at proper failure sites even for initially isotropic material $[11,12]$. Lemaitre and Chaboche [13] firstly generalized isotropic damage mechanics to anisotropic damage mechanics by defining a fourth order tensor. Murakami [14] used a second order tensor to denote damage variable, in consideration of that damage is intrinsically related to the plastic deformation which can be described by a second order tensor. Voyiadjis et al. [15] recently proposed several new anisotropic damage tensors and verified their validity. Although anisotropic damage can be described theoretically by these methods, it is problematic when used in engineering problems. In the study of Zhang and Zhao [16], a truss micro- structure model was proposed to describe the anisotropic damage of material in a simple way, but the value of Poisson's ratio $v$ is a constant of 0.25 . Recently, a boom-panel model was proposed to release the restrictions on the Poisson's ratio $[17,18]$, however, the Poisson's ratio is limited to the range of $0 \sim 0.25$.

In this study, we construct a new anisotropic damage model, i.e. the series model, which removes the restrictions on Poisson's ratio meanwhile possesses the simplicity of describing the anisotropic damage of materials. We couple the truss microstructure, which has the constant Poisson's ratio of 0.25 , in series with an isotropic volumetric elastic element subjected to the same stress tensor $\sigma_{i j}$. This thought is inspired from the study of Caner and Bažant [19] and Voyiadjis [20]. The series model can simulate the material with any value of Poisson's ratio by setting different Poisson's ratio of the coupled isotropic volumetric elastic element. For example, when we set the value of Poisson's ratio of the coupled elastic element by -1 , the Poisson's ratio of the series model will be in the range of -1 0.25; when we set the value of Poisson's ratio of the coupled elastic element by 0.5 , the Poisson's ratio of the series model will be in the range of $0.25 \sim 0.5$. On the other hand, the anisotropy of damage in the material is mainly illustrated by the truss microstructure model; therefore, the simplicity of describing the anisotropic damage is maintained in the series model. Further discussion indicates that at least two independent scalars are needed to characterize the isotropic damage.

\section{Review of the truss microstructure model}

In the study of Zhang and Zhao [16], a truss model is used to simulate a representative volume element (RVE) which is a continuum cubic volume element with edge length $2 l$. The truss microstructure model is shown in Fig. 1, in which every edge or diagonal edge represents one rod that can only resist an axial force. The constitutive relations of the edge rod and the diagonal rod with damage are expressed as follows:

$$
\begin{aligned}
& N_{e}=k_{e} \varphi_{e} \Delta_{e}=k_{e}\left(1-D_{e}\right) \Delta_{e}=K_{e} l\left(1-D_{e}\right) \Delta_{e} ; \\
& N_{d}=k_{d} \varphi_{d} \Delta_{d}=k_{d}\left(1-D_{d}\right) \Delta_{d}=K_{d} l\left(1-D_{d}\right) \Delta_{d},
\end{aligned}
$$

where $k_{e}$ and $k_{d}$ are the stiffness values of the edge rod and the diagonal rod without damage, respectively; $\varphi_{e}$ and $\varphi_{d}$ are the continuity extents of the edge rod and the diagonal rod, respectively; $D_{e}$ and $D_{d}$ are the damage extents of the edge rod and the diagonal rod, respectively; $N_{e}$ and $N_{d}$ are 
the axial forces of the edge rod and the diagonal rod, respectively.

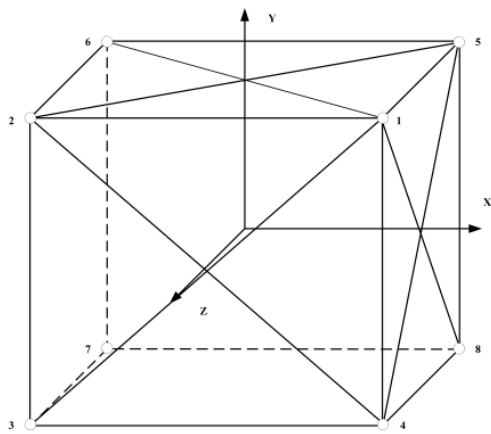

Fig. 1 The truss microstructure

The deformation of the truss microstructure in terms of strains of the RVE is expressed as follows:

$$
\begin{aligned}
& \Delta_{12}=\Delta_{34}=\Delta_{56}=\Delta_{78}=2 l \varepsilon_{x x}^{T} ; \\
& \left.\Delta_{14}=\Delta_{23}=\Delta_{67}=\Delta_{58}=2 l \varepsilon_{y y}^{T} ;\right\} \\
& \Delta_{15}=\Delta_{26}=\Delta_{37}=\Delta_{48}=2 l \varepsilon_{z z}^{T} ; \text {; } \\
& \Delta_{13}=\Delta_{57}=\sqrt{2} l\left(\varepsilon_{x x}^{T}+\varepsilon_{y y}^{T}+2 \varepsilon_{x y}^{T}\right) ; \\
& \Delta_{24}=\Delta_{68}=\sqrt{2} l\left(\varepsilon_{x x}^{T}+\varepsilon_{y y}^{T}-2 \varepsilon_{x y}^{T}\right) \text {; } \\
& \Delta_{18}=\Delta_{27}=\sqrt{2} l\left(\varepsilon_{y y}^{T}+\varepsilon_{z z}^{T}+2 \varepsilon_{y z}^{T}\right) ; \\
& \Delta_{45}=\Delta_{36}=\sqrt{2} l\left(\varepsilon_{y y}^{T}+\varepsilon_{z z}^{T}-2 \varepsilon_{y z}^{T}\right) \text {; } \\
& \Delta_{16}=\Delta_{47}=\sqrt{2} l\left(\varepsilon_{x x}^{T}+\varepsilon_{z z}^{T}+2 \varepsilon_{x z}^{T}\right) \text {; } \\
& \Delta_{25}=\Delta_{38}=\sqrt{2} l\left(\varepsilon_{x x}^{T}+\varepsilon_{z z}^{T}-2 \varepsilon_{x z}^{T}\right),
\end{aligned}
$$

where $\Delta_{k l}$ is the elongation of the rod of NO.kl; and $\Delta_{k l p q}$ is the displacement caused by the shear of panel of NO.klpq; and $\varepsilon_{i j}$ with the superscript $T$ refer to the strain component of the RVE simulated by the truss microstructure.

In addition, the constitutive relation of the RVE with damage is described in terms of the continuity extents and the stiffness of the truss microstructure as follows:

$$
\begin{aligned}
\sigma_{x x} & =\left[2 K_{e} \varphi_{12}+\frac{1}{2} K_{d}\left(\varphi_{13}+\varphi_{24}+\varphi_{16}+\varphi_{25}\right)\right] \varepsilon_{x x}^{T}+ \\
& +\frac{1}{2} K_{d}\left(\varphi_{13}+\varphi_{24}\right) \varepsilon_{y y}^{T}+\frac{1}{2} K_{d}\left(\varphi_{16}+\varphi_{25}\right) \varepsilon_{z z}^{T}+ \\
& +K_{d}\left(\varphi_{13}-\varphi_{24}\right) \varepsilon_{x y}^{T}+K_{d}\left(\varphi_{16}-\varphi_{25}\right) \varepsilon_{x z}^{T} ; \\
\sigma_{y y} & =\left[2 K_{e} \varphi_{14}+\frac{1}{2} K_{d}\left(\varphi_{13}+\varphi_{24}+\varphi_{18}+\varphi_{45}\right)\right] \varepsilon_{y y}^{T}+ \\
& +\frac{1}{2} K_{d}\left(\varphi_{13}+\varphi_{24}\right) \varepsilon_{x x}^{T}+\frac{1}{2} K_{d}\left(\varphi_{18}+\varphi_{45}\right) \varepsilon_{z z}^{T}+ \\
& +K_{d}\left(\varphi_{13}-\varphi_{24}\right) \varepsilon_{x y}^{T}+K_{d}\left(\varphi_{18}-\varphi_{45}\right) \varepsilon_{y z}^{T} ; \\
\sigma_{z z} & =\left[2 K_{e} \varphi_{15}+\frac{1}{2} K_{d}\left(\varphi_{18}+\varphi_{45}+\varphi_{16}+\varphi_{25}\right)\right] \varepsilon_{z z}^{T}+ \\
& +\frac{1}{2} K_{d}\left(\varphi_{16}+\varphi_{25}\right) \varepsilon_{x x}^{T}+\frac{1}{2} K_{d}\left(\varphi_{18}+\varphi_{45}\right) \varepsilon_{y y}^{T}+ \\
& +K_{d}\left(\varphi_{18}-\varphi_{45}\right) \varepsilon_{y z}^{T}+K_{d}\left(\varphi_{16}-\varphi_{25}\right) \varepsilon_{x z}^{T} ;
\end{aligned}
$$

$$
\left.\begin{array}{l}
\sigma_{y z}=\frac{1}{2} K_{d}\left(\varphi_{18}-\varphi_{45}\right)\left(\varepsilon_{z z}^{T}+\varepsilon_{y y}^{T}\right)+K_{d}\left(\varphi_{18}+\varphi_{45}\right) \varepsilon_{y z}^{T} ; \\
\sigma_{x z}=\frac{1}{2} K_{d}\left(\varphi_{16}-\varphi_{25}\right)\left(\varepsilon_{x x}^{T}+\varepsilon_{z z}^{T}\right)+K_{d}\left(\varphi_{16}+\varphi_{25}\right) \varepsilon_{x z}^{T} ; \\
\sigma_{x y}=\frac{1}{2} K_{d}\left(\varphi_{13}-\varphi_{24}\right)\left(\varepsilon_{x x}^{T}+\varepsilon_{y y}^{T}\right)+K_{d}\left(\varphi_{13}+\varphi_{24}\right) \varepsilon_{x y}^{T}
\end{array}\right\}
$$

where

$$
\left.\begin{array}{l}
K_{e}=\frac{E}{5} \\
K_{d}=\frac{2 E}{5}
\end{array}\right\}
$$

where $E$ is the elastic modulus of the RVE.

It should be mentioned that the truss microstructure can only simulate the RVE with the Poisson's ratio $v=0.25$ that is the deficiency of the truss microstructure model.

\section{The series model}

\subsection{Model construction method}

As aforementioned, the truss microstructure is simple and efficient in describing the anisotropic damage of materials, but it can only simulate the material with a Poisson's ratio of 0.25 that would not suffice for most metals. We try to find a way to establish a new model that can simulate most metals meanwhile preserve the advantages of the truss microstructure. From the study of Caner and Bažant [19] and Voyiadjis [20], we are inspired to construct a series model by coupling the truss microstructure in series with an isotropic volumetric elastic element having two elastic constants $E^{C}$ and $v^{C}$. The model is shown in Fig. 2.

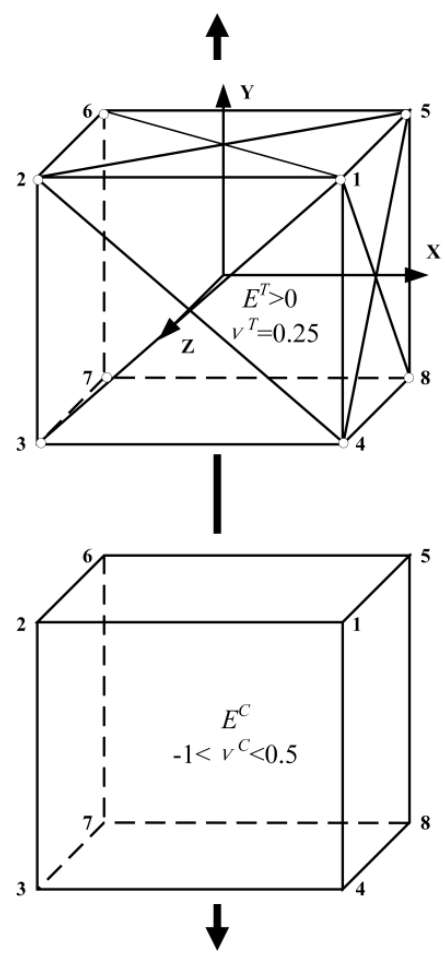

Fig. 2 The series model constructed by coupling the truss microstructure with an isotropic volumetric elastic element 
lows:

The total strain of the RVE is expressed as fol-

$$
\varepsilon_{i j}=\varepsilon_{i j}^{T}+\varepsilon_{i j}^{C},
$$

where $\varepsilon_{i j}$ with the superscript $T$ and $C$ refer to the strain components of the material element simulated by the truss microstructure and the coupled elastic element, respectively.

The constitutive relationship of the RVE can be expressed as follows:

$$
\sigma_{i j}=E_{i j k m} \varepsilon_{k m}
$$

Substituting Eq. (8) into Eq. (9), we can obtain:

$$
\begin{aligned}
\sigma_{i j} & =E_{i j k m}\left(\varepsilon_{k m}^{T}+\varepsilon_{k m}^{C}\right)=E_{i j k m}\left(C_{k m s t}^{T} \sigma_{s t}+C_{k m s t}^{C} \sigma_{s t}\right)= \\
& =E_{i j k m}\left(C_{k m s t}^{T}+C_{k m s t}^{C}\right) \sigma_{s t},
\end{aligned}
$$

where $C_{k m s t}^{T}$ and $C_{k m s t}^{C}$ are the compliance matrixes of the material element simulated by the truss microstructure and the coupled elastic element, respectively.

By using the Voigt notation, and from Eq. (10), yields:

$$
C_{i j k m}=E_{i j k m}^{-1}=C_{k m i j}^{T}+C_{k m i j}^{C} .
$$
as follows:

In the undamaged state, Eq. (11)can be rewritten

$$
C_{0}=E_{0}^{-1}=C_{0}^{T}+C_{0}^{C} .
$$

The undamaged compliance matrix of the isotropic material can be expressed as follows:

$$
C_{0}^{m}=\frac{1}{E^{m}}\left[\begin{array}{cccccc}
1 & -v^{m} & -v^{m} & 0 & 0 & 0 \\
& 1 & -v^{m} & 0 & 0 & 0 \\
& & 1 & 0 & 0 & 0 \\
& & & 2\left(1+v^{m}\right) & 0 & 0 \\
& & & & 2\left(1+v^{m}\right) & 0 \\
& & & & & 2\left(1+v^{m}\right)
\end{array}\right],
$$

where the subscript 0 is attached in $C_{0}^{m}$ to denote the original (initial, undamaged) values of $C^{m}$, and $m$ can be replaced by $T$ or $C$ to denote the compliance matrix $C^{T}$ or $C^{C}$, respectively.

Substitute Eq. (13) into Eq. (12), yields:

$$
\left.\begin{array}{l}
E^{C}=\frac{E\left(4 v^{C}-1\right)}{4 v-1} ; \\
E^{T}=\frac{E\left(4 v^{C}-1\right)}{4\left(v^{C}-v\right)},
\end{array}\right\}
$$

where $E$ and $v$ are the elastic modulus and Poisson's ratio of the material, respectively. Because $E^{T}$ and $E^{C}$ must be nonnegative, we can derive:

$$
\left.\begin{array}{l}
v^{C}>v>0.25 \\
\text { or } \\
v^{C}<v<0.25
\end{array}\right\} \text {. }
$$

From Eq. (15), it is clear that as long as the value of $v^{C}$ is greater than the value of $v$, Poisson's ratio $v$ of the series model will be in the range of $0.25 \sim v^{C}$. For example, if $v^{C}$ is taken as 0.5 , Poisson's ratio $v$ of the series model will be in the range of $0.25 \sim 0.5$. This range covers most possible value of Poisson's ratio of metals. Substitute the value of $v^{C}$ into Eq. (14), yields:

$$
\left.\begin{array}{l}
E_{1}^{C}=\frac{E}{4 v-1} ; \\
E_{1}^{T}=\frac{E}{2(1-2 v)} .
\end{array}\right\}
$$

Similarly, if $v^{C}$ is taken as -1 , the Poisson's ratio $v$ of the series model will be in the range of $-1 \sim 0.25$. Substitute the value of $v^{C}$ into Eq. (14), yields:

$$
\left.\begin{array}{l}
E_{2}^{C}=\frac{5 E}{1-4 v} ; \\
E_{2}^{T}=\frac{5 E}{4(v+1)} .
\end{array}\right\}
$$

Therefore, the series model constructed by coupling the truss microstructure in series with an isotropic elastic element having a Poisson's ratio of -1 or 0.5 can simulate materials with any thermodynamically admissible value of Poisson's ratio. And the series model we are talking in the following sections is limited to that coupled with an isotropic elastic element having a Poisson's ratio of -1 or 0.5 .

\subsection{The damage characteristic of the series model}

3.2.1. The damage characteristic of the truss microstructure

According to Eq. (5)and Eq. (6), the damaged elastic stiffness matrix $\boldsymbol{E}_{\boldsymbol{D}}$ of the truss microstructure can be obtained as follows: 


$$
\boldsymbol{E}_{\boldsymbol{D}}=\left[\begin{array}{cccccc}
E_{x x x x} & E_{x x y y} & E_{x x z z} & 0 & E_{x x x z} & E_{x x x y} \\
& E_{y y y y} & E_{y y z z} & E_{y y y z} & 0 & E_{x x x y} \\
& & E_{z z z z} & E_{y y y z} & E_{x x x z} & 0 \\
& & & E_{y z y z} & 0 & 0 \\
& S y s & & & E_{x z x z} & 0 \\
& & & & & E_{x y x y}
\end{array}\right] \text {, }
$$

where,

$$
\begin{aligned}
& E_{x x x x}=2 K_{e} \varphi_{12}+1 / 2 K_{d}\left(\varphi_{13}+\varphi_{24}+\varphi_{16}+\varphi_{25}\right) ; \\
& E_{x x y y}=1 / 2 K_{d}\left(\varphi_{13}+\varphi_{24}\right) ; \\
& E_{x x z z}=1 / 2 K_{d}\left(\varphi_{16}+\varphi_{25}\right) ; \\
& E_{x x x z}=1 / 2 K_{d}\left(\varphi_{16}-\varphi_{25}\right) ; \\
& E_{x x x y}=1 / 2 K_{d}\left(\varphi_{13}-\varphi_{24}\right) ; \\
& E_{y y y y}=2 K_{e} \varphi_{14}+1 / 2 K_{d}\left(\varphi_{13}+\varphi_{24}+\varphi_{18}+\varphi_{45}\right) ; \\
& E_{\mathrm{yyzz}}=1 / 2 K_{d}\left(\varphi_{18}+\varphi_{45}\right) ; \\
& E_{y y y z}=1 / 2 K_{d}\left(\varphi_{18}-\varphi_{45}\right) ; \\
& E_{z z z z}=2 K_{e} \varphi_{15}+1 / 2 K_{d}\left(\varphi_{18}+\varphi_{45}+\varphi_{16}+\varphi_{25}\right) ; \\
& E_{\mathrm{yzyz}}=K_{d}\left(\varphi_{18}+\varphi_{45}\right) ; \\
& E_{x z x z}=K_{d}\left(\varphi_{16}+\varphi_{25}\right) ; \\
& E_{\mathrm{xyxy}}=K_{d}\left(\varphi_{13}+\varphi_{24}\right) .
\end{aligned}
$$

It is clear from Eq. (19), the damage state of the

$$
C^{C}=\frac{1}{E^{C}}\left[\begin{array}{cc}
\frac{1}{\left(1-D_{11}^{C}\right)^{2}} & \frac{-v^{C}}{\left(1-D_{11}^{C}\right)\left(1-D_{22}^{C}\right)} \\
\\
\\
S y s
\end{array}\right.
$$

where

$$
\left.\begin{array}{l}
G_{23}^{C}=\frac{2\left(1+v^{C}\right)}{\left(1-D_{22}^{C}\right)\left(1-D_{33}^{C}\right)} ; \\
G_{31}^{C}=\frac{2\left(1+v^{C}\right)}{\left(1-D_{33}^{C}\right)\left(1-D_{11}^{C}\right)} ; \\
G_{12}^{C}=\frac{2\left(1+v^{C}\right)}{\left(1-D_{11}^{C}\right)\left(1-D_{22}^{C}\right)},
\end{array}\right\}
$$

material element simulated by the truss microstructure can be represented by the damage extents of the twelve edge rods and twelve diagonal rods. Although the damage of each rod is describe by a single variable, the whole structure exhibits an anisotropic damage property due to the following two reasons: 1) the edge rods of different directions have different damage evolution rates due to different normal stress or strain histories in different directions, which results in different degradations of the elastic stiffness in different directions; 2) the diagonal rods of different planes also have different damage evolution rates because different shear stress or strain histories in different planes, which causes different reductions of the shear stiffness in different planes.

\subsubsection{The damage characteristic of the truss microstructure}

For the series model with $v^{C}$ being taken to be 0.5 , the coupled elastic element has an infinite bulk modulus $K^{C}$, it is reasonable to assume that the element does not change its volume during the damage process, i.e.

$$
\varepsilon_{11}^{C}+\varepsilon_{22}^{C}+\varepsilon_{33}^{C}=0
$$

The damage property of the coupled elastic element can be revealed by the anisotropic damage model of Chow and Wang [21]. In their model, the effective compliance matrix for the coupled elastic element in the principle coordinate system can be expressed as follows:

$$
\left.\begin{array}{cccc}
\frac{-v^{C}}{\left(1-D_{11}^{C}\right)\left(1-D_{33}^{C}\right)} & 0 & 0 & 0 \\
\frac{-v^{C}}{\left(1-D_{22}^{C}\right)\left(1-D_{33}^{C}\right)} & 0 & 0 & 0 \\
\frac{1}{\left(1-D_{33}^{C}\right)^{2}} & 0 & 0 & 0 \\
& G_{23}^{C} & 0 & 0 \\
& & G_{31}^{C} & 0 \\
& & & G_{12}^{C}
\end{array}\right],
$$

and $D_{11}^{\mathrm{C}}, D_{22}^{C}$ and $D_{33}^{C}$ are the damage variables at their respective principle axes. In the principle coordinate system, the stress is denoted as:

$$
\left\{\boldsymbol{\sigma}^{T}\right\}=\left\{\sigma_{11}^{C}, \sigma_{22}^{C}, \sigma_{33}^{C}, 0,0,0\right\} .
$$

We can rewrite Eq. (20) in terms of stress as follows:

$$
\begin{aligned}
& {\left[\frac{1}{\left(1-D_{11}^{C}\right)^{2}}-\frac{v^{C}}{\left(1-D_{11}^{C}\right)\left(1-D_{22}^{C}\right)}-\frac{v^{C}}{\left(1-D_{11}^{C}\right)\left(1-D_{33}^{C}\right)}\right] \sigma_{11}^{C}+\left[\frac{1}{\left(1-D_{22}^{C}\right)^{2}}-\frac{v^{C}}{\left(1-D_{11}^{C}\right)\left(1-D_{22}^{C}\right)}-\frac{v^{C}}{\left(1-D_{22}^{C}\right)\left(1-D_{33}^{T}\right)}\right] \sigma_{22}^{C}+} \\
& +\left[\frac{1}{\left(1-D_{33}^{C}\right)^{2}}-\frac{v^{C}}{\left(1-D_{11}^{C}\right)\left(1-D_{33}^{C}\right)}-\frac{v^{C}}{\left(1-D_{22}^{C}\right)\left(1-D_{33}^{C}\right)}\right] \sigma_{33}^{C}=0 .
\end{aligned}
$$


Because $\sigma_{11}^{C}, \sigma_{22}^{C}$ and $\sigma_{33}^{C}$ are independent of each other, the coefficients of $\sigma_{11}^{C}, \sigma_{22}^{C}$ and $\sigma_{33}^{C}$ must be equal to 0 , that is:

$$
\left.\begin{array}{l}
\frac{1}{\left(1-D_{11}^{C}\right)^{2}}-\frac{v^{C}}{\left(1-D_{11}^{C}\right)\left(1-D_{22}^{C}\right)}-\frac{v^{C}}{\left(1-D_{11}^{C}\right)\left(1-D_{33}^{C}\right)}=0 ; \\
\frac{1}{\left(1-D_{22}^{C}\right)^{2}}-\frac{v^{C}}{\left(1-D_{11}^{C}\right)\left(1-D_{22}^{C}\right)}-\frac{v^{C}}{\left(1-D_{22}^{C}\right)\left(1-D_{33}^{T}\right)}=0 ; \\
\frac{1}{\left(1-D_{33}^{C}\right)^{2}}-\frac{v^{C}}{\left(1-D_{11}^{C}\right)\left(1-D_{33}^{C}\right)}-\frac{v^{C}}{\left(1-D_{22}^{C}\right)\left(1-D_{33}^{C}\right)}=0 .
\end{array}\right\}
$$

It is clear that $D_{11}^{\mathrm{C}}, D_{22}^{C}$ and $D_{33}^{C}$ should be equal to each other, that is:

$$
D_{11}^{C}=D_{22}^{C}=D_{33}^{C}=D_{1}^{C},
$$

which indicates the damage of the coupled elastic element is isotropic.

Let $v^{C}$ be equal to 0.5 and substitute Eq. (26) into Eq. (21), we have the damaged elastic compliance matrix of the coupled elastic element as follows:

$$
C_{1}^{C}=\frac{1}{E_{1}^{C}\left(1-D_{1}^{C}\right)^{2}}\left[\begin{array}{crrrrr}
1 & -\frac{1}{2} & -\frac{1}{2} & 0 & 0 & 0 \\
1 & -\frac{1}{2} & 0 & 0 & 0 \\
& 1 & 0 & 0 & 0 \\
S y s & & & 3 & 0 & 0 \\
& & & & & 3
\end{array}\right] .
$$

For the series model with $v^{C}$ being taken to be -1 , the coupled elastic element has an infinite shear modulus $G^{C}$, it is reasonable to assume that the element does not change its shape during the damage process, i.e. $v^{C}$ is always equal to -1 during the damage process. For the case of uniaxial tension, the principle strains of coupled elastic element can be written as follows:

$$
\left.\begin{array}{l}
\varepsilon_{11}^{C}=\frac{\sigma_{11}^{C}}{E^{C}\left(1-D_{11}^{C}\right)^{2}} ; \\
\varepsilon_{22}^{C}=\frac{\sigma_{11}^{C}}{E^{C}\left(1-D_{11}^{C}\right)\left(1-D_{22}^{C}\right)} ; \\
\varepsilon_{33}^{C}=\frac{\sigma_{11}^{C}}{E^{C}\left(1-D_{11}^{C}\right)\left(1-D_{33}^{C}\right)} .
\end{array}\right\}
$$

Under the assumption that $v^{C}$ does not change, the principle strains in three directions should be identical, then we can deduce that:

$$
D_{11}^{C}=D_{22}^{C}=D_{33}^{C}=D_{2}^{C},
$$

which means the damage of the coupled elastic element with Poisson's ratio of -1 is also isotropic. Take $v^{C}=-1$ into consideration and substitute Eq. (29)into Eq. (21), we obtain the damaged elastic compliance matrix of the coupled elastic element as follows:

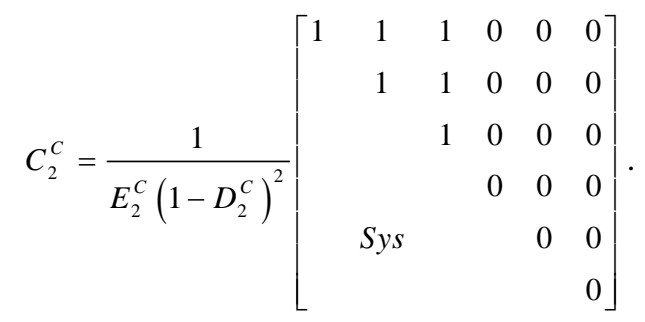

\section{Damage evolution equation}

\subsection{Strain energy density of the series model}

The Helmholtz free energy taken as the state potential of the material is a function of all the state variables [22], which can be expressed in the form in the present elastic context:

$$
\psi=\psi_{e}=\psi_{e}\left(\boldsymbol{\varepsilon}^{e}, \boldsymbol{D}, T\right),
$$

where $T$ is the absolute temperature. The thermodynamic variables corresponding to the elastic strain tensor $\boldsymbol{\varepsilon}^{e}$ and the damage tensor $\boldsymbol{D}$ are:

$$
\begin{gathered}
\sigma_{i j}=\rho \frac{\partial \psi_{e}}{\partial \varepsilon_{i j}^{e}} \\
Y_{i j}=-\rho \frac{\partial \psi_{e}}{\partial D_{i j}},
\end{gathered}
$$

where $\rho$ is the mass density. The latter variable $Y$, which is denoted as the thermodynamic conjugate force, turns out to have the same physical significance as the strain energy release rate of fracture mechanics.

For linear isothermal elasticity, the free energy is given in terms of the strain energy density $\omega_{e}$ as:

$$
\rho \psi_{e}=\omega_{e}
$$

The strain energy density of the RVE can be expressed as: 


$$
\begin{aligned}
\omega_{e}= & \frac{W_{e}}{V}=\frac{\iiint \frac{\sigma_{i j} \varepsilon_{i j}}{2}}{8 l^{3}}=\frac{\iiint \frac{\sigma_{i j}\left(\varepsilon_{i j}^{T}+\varepsilon_{i j}^{C}\right)}{2}}{8 l^{3}}= \\
= & \frac{W_{e}^{T}+W_{e}^{C}}{8 l^{3}}=\omega_{e}^{T}+\omega_{e}^{C},
\end{aligned}
$$

where $W_{e}^{T}$ and $W_{e}^{C}$ denote the strain energy of the truss microstructure and the coupled elastic volume element, respectively. $W_{e}^{T}$ can be expressed by the summation of the strain energy of the rods.

$$
\begin{aligned}
& W_{e}^{T}=4\left(W_{12}^{T}+W_{14}^{T}+W_{15}^{T}\right)+ \\
& +2\left(W_{13}^{T}+W_{16}^{T}+W_{18}^{T}+W_{24}^{T}+W_{25}^{T}+W_{45}^{T}\right) . \\
& \omega_{e}^{T}=K_{e}\left[\left(1-D_{12}\right)\left(\varepsilon_{12}^{T}\right)^{2}+\left(1-D_{14}\right)\left(\varepsilon_{14}^{T}\right)^{2}+\left(1-D_{15}\right)\left(\varepsilon_{15}^{T}\right)^{2}\right]+K_{d}\left[\left(1-D_{13}\right)\left(\varepsilon_{13}^{T}\right)^{2}+\left(1-D_{24}\right)\left(\varepsilon_{24}^{T}\right)^{2}+\left(1-D_{18}\right)\left(\varepsilon_{18}^{T}\right)^{2}\right]+ \\
& +K_{d}\left[\left(1-D_{45}\right)\left(\varepsilon_{45}^{T}\right)^{2}+\left(1-D_{16}\right)\left(\varepsilon_{16}^{T}\right)^{2}+\left(1-D_{25}\right)\left(\varepsilon_{25}^{T}\right)^{2}\right] \text {. } \\
& \omega_{e, 1}^{C}=\frac{\sigma_{i j}^{C} \varepsilon_{i j}^{C}}{2}=\frac{\sigma_{11}^{C} \varepsilon_{11}^{C}+\sigma_{22}^{C} \varepsilon_{22}^{C}+\sigma_{33}^{C} \varepsilon_{33}^{C}}{2}= \\
& =\frac{E_{1}^{C}\left(1-D_{1}^{C}\right)^{2}}{3}\left[\left(\varepsilon_{11}^{C}\right)^{2}+\left(\varepsilon_{22}^{C}\right)^{2}+\left(\varepsilon_{33}^{C}\right)^{2}\right] \text {. }
\end{aligned}
$$

For the coupled elastic element with the Poisson's ratio of -1 , the strain energy density $\omega_{e, 2}^{C}$ can be derived as follows:

$$
\begin{aligned}
\omega_{e, 2}^{C} & =\frac{\sigma_{i j}^{C} \varepsilon_{i j}^{C}}{2}=\frac{\sigma_{11}^{C} \varepsilon_{11}^{C}+\sigma_{22}^{C} \varepsilon_{22}^{C}+\sigma_{33}^{C} \varepsilon_{33}^{C}}{2}= \\
& =\frac{E_{2}^{C}\left(1-D_{2}^{C}\right)^{2}}{2}\left(\varepsilon_{11}^{C}\right)^{2} .
\end{aligned}
$$

\subsection{Damage evolution law}

According to the law of thermodynamics, the damage evolution law can be obtained from a dissipation function $\varphi^{*}$, which is a convex function of the associated variables:

$$
\varphi^{*}=\varphi^{*}(\varepsilon, \dot{\boldsymbol{D}}, T, \boldsymbol{D}) .
$$

Using Legendre's Fenchel transformation, an equivalent dual dissipation potential can be obtained:

$$
\varphi=\varphi(\dot{\boldsymbol{Y}}, \boldsymbol{Y}, \boldsymbol{D}, \boldsymbol{\sigma}, T),
$$

then the damage evolution law can be expressed as[4]:

$$
\dot{D}_{i j}=\lambda \frac{\partial \varphi}{\partial Y_{i j}},
$$

where $W_{12}^{T}, W_{14}^{T}$, and $W_{15}^{T}$ are the strain energy of the edge rods of No. 12, 14, and 15, respectively. And $W_{13}^{T}$, $W_{16}^{T}, W_{18}^{T}, W_{24}^{T}, W_{25}^{T}$ and $W_{45}^{T}$ are the strain energy of the diagonal rods of No. 13, 16, 18, 24, 25, and 45, respectively. And take $W_{12}^{T}$ as an example, it can be deduced as follows:

$$
\begin{aligned}
W_{12}^{T} & =\frac{N_{12} \Delta_{12}}{2}=\frac{K_{e} l\left(1-D_{12}\right) \Delta_{12}^{2}}{2}= \\
& =\frac{K_{e} l\left(1-D_{12}\right)\left(2 l \varepsilon_{12}^{T}\right)^{2}}{2}=2 K_{e} l^{3}\left(1-D_{12}\right)\left(\varepsilon_{12}^{T}\right)^{2} .
\end{aligned}
$$

Similarly, we can deduce the strain energy of the other rods, and substitute them into Eq. (36), we can write $\omega_{e}^{T}$ as follows: where $\lambda$ is a multiplier defined from the damage criterion.

Then by defining proper dissipation potential and damage criterion, we can obtain the damage evolution law.

According to Eq. (33)and Eq. (38), the thermodynamic conjugate force corresponding to the damage extent $D_{12}$ can be derived as follows:

$$
Y_{12}=-\rho \frac{\partial \psi_{e}}{\partial D_{12}}=-\frac{\partial \omega_{e}}{\partial D_{12}}=-\frac{\partial \omega_{e}^{T}}{\partial D_{12}}=K_{e}\left(\varepsilon_{12}^{T}\right)^{2}
$$

Similarly, the thermodynamic conjugate forces corresponding to other damage extents can also be obtained as follows:

$$
\left.\begin{array}{c}
Y_{i j}=K_{e}\left(\varepsilon_{i j}^{T}\right)^{2} ; \\
Y_{s t}=K_{d}\left(\varepsilon_{s t}^{T}\right)^{2},
\end{array}\right\}
$$

where the subscript $i j$ represents the number of the edge rod, such as 12, 14, and 15; the subscript st represents the number of the diagonal rod, such as $13,16,18,24,25$, and 45.

According to Eq. (33)and Eq. (38), we can obtain the thermodynamic conjugate force of $D_{1}^{C}$ as follows:

$$
\begin{aligned}
Y_{1}^{C} & =-\rho \frac{\partial \psi_{e}}{\partial D_{1}^{C}}=-\frac{\partial \omega_{e}}{\partial D_{1}^{C}}=-\frac{\partial \omega_{e, 1}^{C}}{\partial D_{1}^{C}}= \\
& =\frac{2}{3} E_{1}^{C}\left(1-D_{1}^{C}\right)\left[\left(\varepsilon_{11}^{C}\right)^{2}+\left(\varepsilon_{22}^{C}\right)^{2}+\left(\varepsilon_{33}^{C}\right)^{2}\right] .
\end{aligned}
$$

Similarly, we can obtain the thermodynamic conjugate force of $D_{2}^{C}$ as follows:

$$
Y_{2}^{C}=-\rho \frac{\partial \psi_{e}}{\partial D_{2}^{C}}=-\frac{\partial \omega_{e}}{\partial D_{2}^{C}}=-\frac{\partial \omega_{e, 2}^{C}}{\partial D_{2}^{C}}=E_{2}^{C}\left(1-D_{2}^{C}\right)\left(\varepsilon_{11}^{C}\right)^{2}
$$


Further, substituting Eqs. (43), (44)and (45) or (46) into Eq. (42), we can obtained the damage evolution equation of all damage variables.

\section{Further discussions on this model}

This construction method of the series model is not only suitable for the truss microstructure coupled by an elastic element, but also can be applied to couple two arbitrary elastic elements in series to construct a continuum damage model.

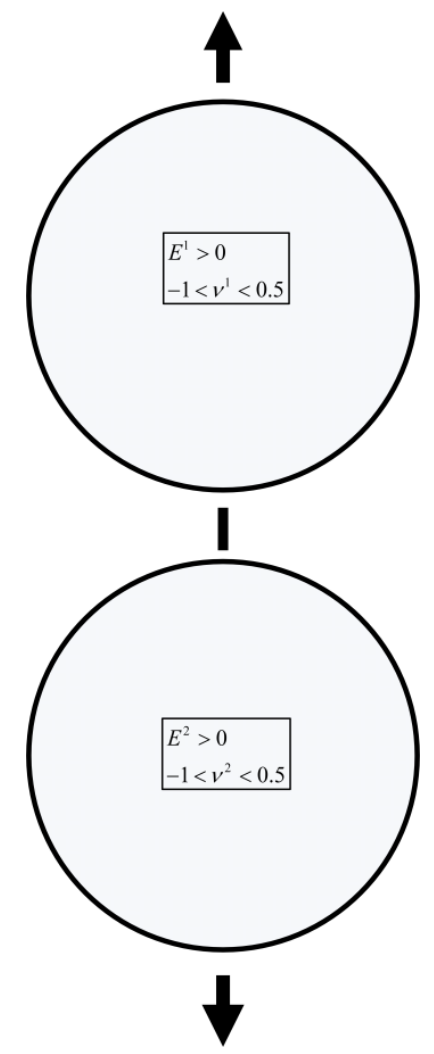

Fig. 3 The general anisotropic damage model

For the most general case, we can couple two continuum volumetric elastic elements in series to construct a general continuum damage model which is shown in Fig. 3. According to Eq. (13) and Eq. (12), the initial elastic constants of two elastic element can be obtained as follows:

1) when $v^{1} \neq v^{2} \neq v$,

$$
\left.\begin{array}{l}
E^{1}=\frac{E\left(v^{2}-v^{1}\right)}{v^{2}-v} ; \\
E^{2}=\frac{E\left(v^{2}-v^{1}\right)}{v-v^{1}},
\end{array}\right\}
$$

where $E^{1}$ and $E^{2}$ are the elastic moduli of two coupled elastic elements, respectively; $v^{1}$ and $v^{2}$ are Poisson's ratios of two coupled elastic elements, respectively; $E$ and $v$ are the elastic modulus and Poisson's ratio of the RVE, respectively. Considering that $E^{1}$ and $E^{2}$ must be nonnegative, we can get:

$$
\left.\begin{array}{l}
v^{2}>v>v^{1} \\
\text { or } \\
v^{1}>v>v^{2}
\end{array}\right\}
$$

2) when $v^{1}=v^{2}=v$,

$$
\frac{1}{E^{1}}+\frac{1}{E^{2}}=\frac{1}{E}
$$

If we take $v^{1}$ and $v^{2}$ to be in the range of $-1 \sim 0.5$, the damage characteristic of the coupled elastic elements is anisotropic, we can get a most general anisotropic damage model. Specially, if we take $v^{1}$ as 0.25 , and take $v^{2}$ as 0.5 or -1 , this model becomes the aforementioned series model by coupling the truss microstructure with an elastic isotropic damage element. Further, if we take $v^{1}$ as 0.5 , and take $v^{2}$ as -1 , the bi-variable isotropic damage model of the most general form can be constructed, which is shown in Fig. 4.

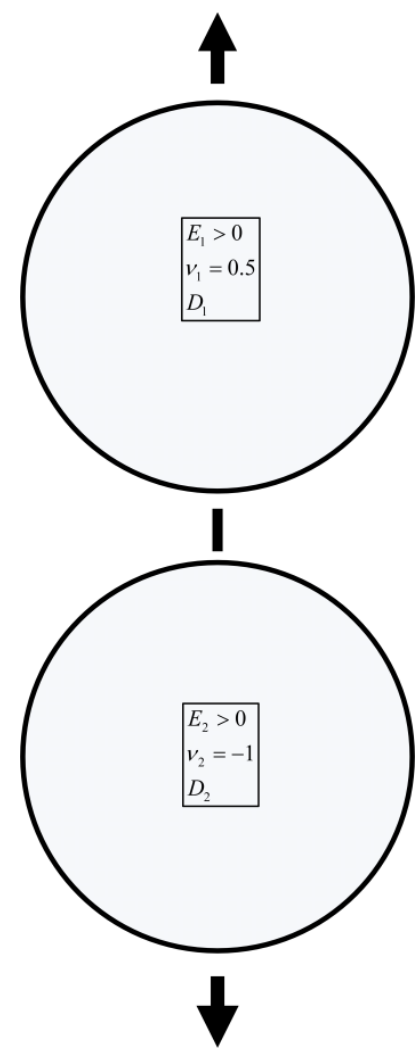

Fig. 4 The bi-variable isotropic damage model

It is obvious that the first elastic element only possesses the distortional strain energy and the second elastic element only includes the strain energy of volumetric deformation. The elastic constants of the two coupled elements can be obtained according to Eq. (47)as follows:

$$
\left.\begin{array}{l}
E^{1}=\frac{3 E}{2(1+v)}=3 G \\
E^{2}=\frac{3 E}{1-2 v}=9 K .
\end{array}\right\}
$$

The corresponding damages of the elastic elements have the physically significant meanings relating to bulk and shear responses, which can be expressed as follows: 


$$
\left.\begin{array}{l}
D^{1}=1-\frac{\tilde{G}}{G} \\
D^{2}=1-\frac{\tilde{K}}{K}
\end{array}\right\}
$$

It should be noted that we cannot derive a scalar isotropic damage model by using this coupling method, the reason of which can be briefly illustrated as follows. The series coupling model is a scalar isotropic damage model only if that the damage property of each of the two elastic elements is isotropic and the damage extents of them are identical, i.e. $D^{1}=D^{2}$. As we mentioned in section "The damage characteristic of the coupled elastic element", only the elastic element with Poisson's ratio of -1 or 0.5 , the damage property is isotropic. Therefore, the only way to derive a scalar isotropic damage model is to couple two elastic elements with the same Poisson's ratio ( -1 or 0.5$)$. However, two elastic elements having the same Poisson's ratio of -1 or 0.5 coupled in series have no practical significance. Thus, we cannot get a scalar isotropic damage model by using this coupling method, which again confirms that two independent scalars are needed to characterize the isotropic damage that discussed by Cauvin and Testa [23].

\section{Conclusions}

In this work, a series model is constructed by coupling the truss microstructure with an elastic element, which can simulate the material with any thermodynamically admissible value of Poisson's ratio as well as retain the simplicity of the truss microstructure. Poisson's ratio of the series model will be in the range of $-1 \sim 0.25$, if Poisson's ratio of the coupled elastic element is taken as -1 , while Poisson's ratio of the series model will be in the range of 0.25 0.5 if Poisson's ratio of the coupled elastic element is taken as 0.5 .

The damage properties of the series model are studied. The basic equations of the general anisotropic damage model are presented, and a bi-variable isotropic model is degenerated from the series model. But we cannot derive a scalar isotropic damage model by means of this coupling method, which confirms that two independent scalars are needed to characterize the isotropic damage. This is only an initial work, more researches on the coupling methods both in series or in parallel will be further carried on.

\section{References}

1. Kachanov, L. 1958. Time of the rupture process under creep conditions, Isv. Akad. Nauk. SSR. Otd Tekh. Nauk 8: 26-31.

2. Murakami, S. 2012. Continuum Damage Mechanics: A Continuum Mechanics Approach to the Analysis of Damage and Fracture, Springer Science \& Business Media. http://dx.doi.org/10.1007/978-94-007-2666-6.

3. Rabotnov, Y.N. 1969. Creep Rupture, Applied mechanics. Springer.

4. Lemaitre, J.; Lippmann, H. 1996. A Course on Damage Mechanics. Springer, Berlin. http://dx.doi.org/10.1007/978-3-642-18255-6.
5. Tang, X.S.; Jiang, C.P,; Zheng, J.L. 2001. General expressions of constitutive equations for isotropic elastic damaged materials, Applied Mathematics and Mechanics 22: 1468-1475. http://dx.doi.org/10.1023/A:1022899129861.

6. Voyiadjis, G.Z.; Kattan, P. 2009. A comparative study of damage variables in continuum damage mechanics, International Journal of Damage Mechanics 18(4): 315-340. http://dx.doi.org/10.1177/1056789508097546.

7. Ju, J. 1990. Isotropic and anisotropic damage variables in continuum damage mechanics, Journal of Engineering Mechanics 116: 2764-2770.

http://dx.doi.org/10.1061/(ASCE)0733-9399(1990)116: 12(2764).

8. Cauvin, A.; Testa, R.B. 1999. Elastoplastic material with isotropic damage, International Journal of Solids and Structures 36: 727-746. http://dx.doi.org/10.1016/S0020-7683(98)00043-2.

9. Tang, C.; Shen, W.; Peng, L.; Lee, T. 2002. Characterization of isotropic damage using double scalar variables, International Journal of Damage Mechanics 11: 3-25.

http://dx.doi.org/10.1106/105678902023194.

10. Lemaitre, J. 1984. How to use damage mechanics, Nuclear Engineering and Design 80: 233-245. http://dx.doi.org/10.1016/0029-5493(84)90169-9.

11. Hao, L.; Ke, P.; June, W. 1985. An anisotropic damage criterion for deformation instability and its application to forming limit analysis of metal plates, Engineering Fracture Mechanics 21: 1031-1054. http://dx.doi.org/10.1016/0013-7944(85)90008-6.

12. Chow, C.; Wang, J. 1987. An anisotropic theory of elasticity for continuum damage mechanics, International Journal of Fracture 33: 3-16. http://dx.doi.org/10.1007/BF00034895.

13. Lemaitre, J.; Chaboche, J.L. 1990 Mechanics of Solid Materials, Cambridge university press, 584 p. http://dx.doi.org/10.1017/CBO9781139167970.

14. Murakami, S. 1988. Mechanical modeling of material damage, Journal of Applied Mechanics 55: 280-286. http://dx.doi.org/10.1115/1.3173673.

15. Voyiadjis, G.Z.; Yousef, M.A.; Kattan, P.I. 2012. New tensors for anisotropic damage in continuum damage mechanics, Journal of Engineering Materials and Technology 134: 0210151-0210157. http://dx.doi.org/10.1115/1.4006067.

16.Zhang, X.; Zhao, J. 1998. Applied fatigue damage mechanics of metallic structural members. Beijing. National Defense Industry Press (in Chinese).

17. Zhang, M.; Meng, Q.C.; Hu, W.P.; Zhang, X. 2012 Study on anisotropic fatigue damage model of metal component, International Journal of Damage Mechanics 21: 599-620. http://dx.doi.org/10.1177/1056789511414216.

18. Sun, L.L.; Hu, W.P.; Zhang, M.; Meng, Q.C. 2014 An anisotropic damage model based on microstructure of boom-panel for the fatigue life prediction of structural components, Fatigue \& Fracture of Engineering Materials \& Structures 37(11): 1186-1196. http://dx.doi.org/10.1111/ffe.12188.

19. Caner, F.C.; Bažant, Z.P. 2012. Microplane model M7 for plain concrete. I: Formulation, Journal of Engi- 
neering Mechanics 139: 1714-1723. http://dx.doi.org/10.1061/(ASCE)EM.1943-7889.00005 70.

20. Voyiadjis, G.Z.; Kattan, P.I. 2012. Mechanics of damage processes in series and in parallel: a conceptual framework. Acta Mechanica 223: 1863-1878. http://dx.doi.org/10.1007/s00707-012-0678-0.

21. Chow, C.L.; Wang, J. 1987. An anisotropic theory of elasticity for continuum damage mechanics, International Journal of Fracture 33: 3-16. http://dx.doi.org/10.1007/BF00034895.

22. Lemaitre, J.; Desmorat, R. 2005. Engineering Damage Mechanics: Ductile, Creep, Fatigue and Brittle Failures. Springer.

23. Cauvin, A.; Testa, R.B. 1999. Damage mechanics: basic variables in continuum theories, International Journal of Solids and Structures 36: 747-761. http://dx.doi.org/10.1016/S0020-7683(98)00044-4.
Linlin Sun, Weiping Hu, Qingchun Meng

AN ANISOTROPIC DAMAGE MODEL OF ELASTICITY BASED ON A SERIES COUPLING MODEL

S u m m a r y

This paper presents the study of an equivalent anisotropic elastic damage model, i.e. the series model, which is constructed by coupling the truss microstructure in series with an isotropic volumetric elastic element. This model can simulate the material with any thermodynamically admissible values of Poisson's ratio as well as inherit the simplicity of the truss microstructure model. Further discussion indicates that at least two independent scalars are needed to characterize the isotropic damage. This coupling method presented in this work provides a new way to research anisotropic damage of materials.

Keywords: constitutive modeling; elasticity; truss microstructure; anisotropic damage; coupling method.

Received May 26, 2015

Accepted January 06, 2016 